\title{
Staged Stereotactic Radiosurgery Decreases Symptomatic Radionecrosis in Large Brain Metastasis
}

\author{
NEIL CHEVLI ${ }^{1}$, HUI-CHUAN WANG ${ }^{2}$, PRACHI DUBEY ${ }^{3}$, WAQAR HAQUE ${ }^{2}$, ANDREW M. FARACH ${ }^{2}$, \\ RAMIRO PINO ${ }^{2}$, ROBERT C. ROSTOMILY ${ }^{4}$, E. BRIAN BUTLER ${ }^{2}$ and BIN S. TEH ${ }^{2}$ \\ ${ }^{1}$ Department of Radiation Oncology, University of Texas Medical Branch at Galveston, Galveston, TX, U.S.A.; \\ ${ }^{2}$ Department of Radiation Oncology, Houston Methodist Hospital, Houston, TX, U.S.A.; \\ ${ }^{3}$ Department of Neuroradiology, Houston Methodist Hospital, Houston, TX, U.S.A.; \\ ${ }^{4}$ Department of Neurosurgery, Houston Methodist Hospital, Houston, TX, U.S.A.
}

\begin{abstract}
Background: Limited brain metastasis is treated definitively with stereotactic radiosurgery when surgical resection is not indicated. Although this has historically been performed in a single fraction, multi-fraction approaches such as fraction radiosurgery (FSRS) and staged radiosurgery (SSRS) have been recently examined as alternative approaches for larger lesions to permit better tumor control without increased toxicity. Case Report: We present the case of a patient who developed symptomatic radionecrosis in two brain metastasis, $2.3 \mathrm{~cm}$ and $2.1 \mathrm{~cm}$ in size, which were treated with 18 Gy in one fraction, but no radionecrosis in a $3.3 \mathrm{~cm}$ lesion treated in two fractions of 15 Gy nor in two punctate lesions that were treated in one fraction of $20 \mathrm{~Gy}$. Although she did not respond to steroids, she responded to bevacizumab symptomatically and on neuroimaging. Conclusion: Congruent with other recent studies, our report suggests that large brain metastasis should be considered for FSRS/SSRS.
\end{abstract}

Standard of care for large or symptomatic brain metastasis is surgical resection followed by stereotactic radiosurgery (SRS) to the resection cavity (1). All other limited brain metastases are treated definitively with SRS (1). Definitive SRS has historically been performed in a single fraction, with dose limited by tumor size in order to minimize toxicity

This article is freely accessible online.

Correspondence to: Bin S. Teh, MD, Houston Methodist Hospital, Department of Radiation Oncology, 6565 Fannin St. DB1-077, Houston, TX 77030, U.S.A. Tel: +1 7134414800, e-mail: bteh@houstonmethodist.org

Key Words: Staged stereotactic radiosurgery, symptomatic radionecrosis, radiographical radionecrosis, large brain metastasis, fractionated stereotactic radiosurgery.
(2). Because tumor control is related to dose, multi-fraction approaches have been proposed for larger lesions to reduce recurrence without increasing toxicity. These include fractionated radiosurgery (FSRS) and staged radiosurgery (SSRS). The former involves daily treatments, most commonly 9 Gy per fraction delivered three consecutive days. The latter involves two fractions separated by about one month, most commonly 15 Gy per fraction.

While national guidelines suggest lesions $>3 \mathrm{~cm}$ be treated with these multi-fraction approaches, in an effort to avoid symptomatic radionecrosis, recent studies have investigated a lower cutoff of $2 \mathrm{~cm}(1,3-4)$. We provide supporting evidence to the lower threshold by reporting a patient who developed symptomatic radionecrosis in two brain metastasis $2.3 \mathrm{~cm}$ and $2.1 \mathrm{~cm}$ in size that were treated with SRS in one fraction, but no radionecrosis in a $3.3 \mathrm{~cm}$ lesion treated in two fractions (SSRS) nor in two punctate lesions that were treated in one fraction. This type of scenario has not previously been described in the literature.

\section{Case Report}

A 44-year-old female palpated a left breast mass. Diagnostic ultrasound showed a $5 \mathrm{~cm}$ left breast mass and biopsy showed grade 2 triple negative invasive ductal carcinoma. She received neoadjuvant chemotherapy with 5-fluorouracil, epirubicin, and cyclophosphamide followed by paclitaxel. At the time of left mastectomy, there was $2 \mathrm{~mm}$ of residual carcinoma with no positive sentinel lymph nodes. She then received 50 Gy in 25 fractions to the left chest wall and regional lymph nodes followed by $10 \mathrm{~Gy}$ in 5 fractions to the mastectomy scar.

Two years after completing therapy, she experienced nodal relapse with involvement of the left subpectoral region, anterior mediastinum, right supraclavicular region, and right cervical region. She received paclitaxel for 9 months, but was then switched to eribulin after experiencing progression 
in the sternum and anterior mediastinum. She developed brain metastasis 3 months later and was referred for stereotactic radiosurgery.

High resolution contrast enhanced MR of the brain demonstrated three lesions- a $3.3 \mathrm{~cm} 29 \mathrm{cc}$ peripheral rimenhancing lesion in the left parasagittal frontoparietal lobe (Figure 1A), a $2.3 \mathrm{~cm} 12 \mathrm{cc}$ lesion in the posterior right frontal lobe (Figure 2A), and a $2.1 \mathrm{~cm} 6 \mathrm{cc}$ lesion in the anterior right frontal lobe (Figure 3A). The latter two lesions were irregularly enhancing with susceptibility artifact on T2-star GRE sequence, suggesting intra-lesional hemorrhage or calcification. These two lesions were treated with stereotactic radiosurgery with a dose of $18 \mathrm{~Gy}$ prescribed to the $60 \%$ isodose line (IDL) in one fraction using the Gamma Knife ${ }^{\circledR}$ Icon. The larger lesion received two fractions of 15 Gy prescribed to the $60 \%$ IDL delivered 39 days apart. MRI prior to the second fraction showed this lesion had decreased to $24 \mathrm{cc}$ volume with decreased rim-enhancement (Figure 1B); the two right frontal lesions had decreased to $<1 \mathrm{~cm}$ in size with progressive blood products within the lesions (Figure $2 \mathrm{~B}$ and $3 \mathrm{~B}$ ).

MRI one month after the second stage showed two new punctate lesions in the bilateral frontal lobes that were each $2 \mathrm{~mm}$ in size with a volume of $0.26 \mathrm{cc}$ and $0.15 \mathrm{cc}$. Additionally, the prior left sided lesion now measured $2.6 \mathrm{~cm}$ (Figure 1C), smaller than prior measurement of $3.3 \mathrm{~cm}$, and the two right sided lesions remained stable (Figure 2C, Figure $3 \mathrm{C}$ ). The two new punctate lesions were treated with SRS to a dose of 20 Gy to the $60 \%$ IDL in one fraction using the Gamma Knife ${ }^{\circledR}$ Icon.

At follow-up one month later, the patient reported new headaches but denied all other neurologic symptoms. Brain MRI at this time showed the left parietal lesion had further decreased to $2.3 \mathrm{~cm}$ in size (Figure 1D). However, the anterior right frontal lesion had increased to $3 \mathrm{~cm}$ in size (Figure 3D) and the posterior right frontal lobe lesion had increased to $1.5 \mathrm{~cm}$ in size (Figure 2D). She was placed on dexamethasone. Brain MRI two months later showed further progression of the two right frontal lesions (Figure 2E and $3 \mathrm{E})$, the largest now measuring $4 \mathrm{~cm}$, and further regression of the left parietal lesion (Figure 1E). At this time, the patient was experiencing syncopal episodes and seizure-like activity in her arms. She was placed on levetiracetam. Imaging was discussed at our institutional multidisciplinary tumor board with consensus that the changes in the right frontal lobe lesions represented radionecrosis refractory to dexamethasone. The patient subsequently received four cycles of bevacizumab.

Brain MRI 2 months later showed marked improvement in all three residual lesions (Figure $1 \mathrm{~F}, 2 \mathrm{~F}$ and $3 \mathrm{~F}$ ). In addition, her syncopal episodes and seizure like activity had resolved. She was successfully weaned off dexamethasone. Brain MRI three months later remained stable with no new metastasis and the patient remained asymptomatic.
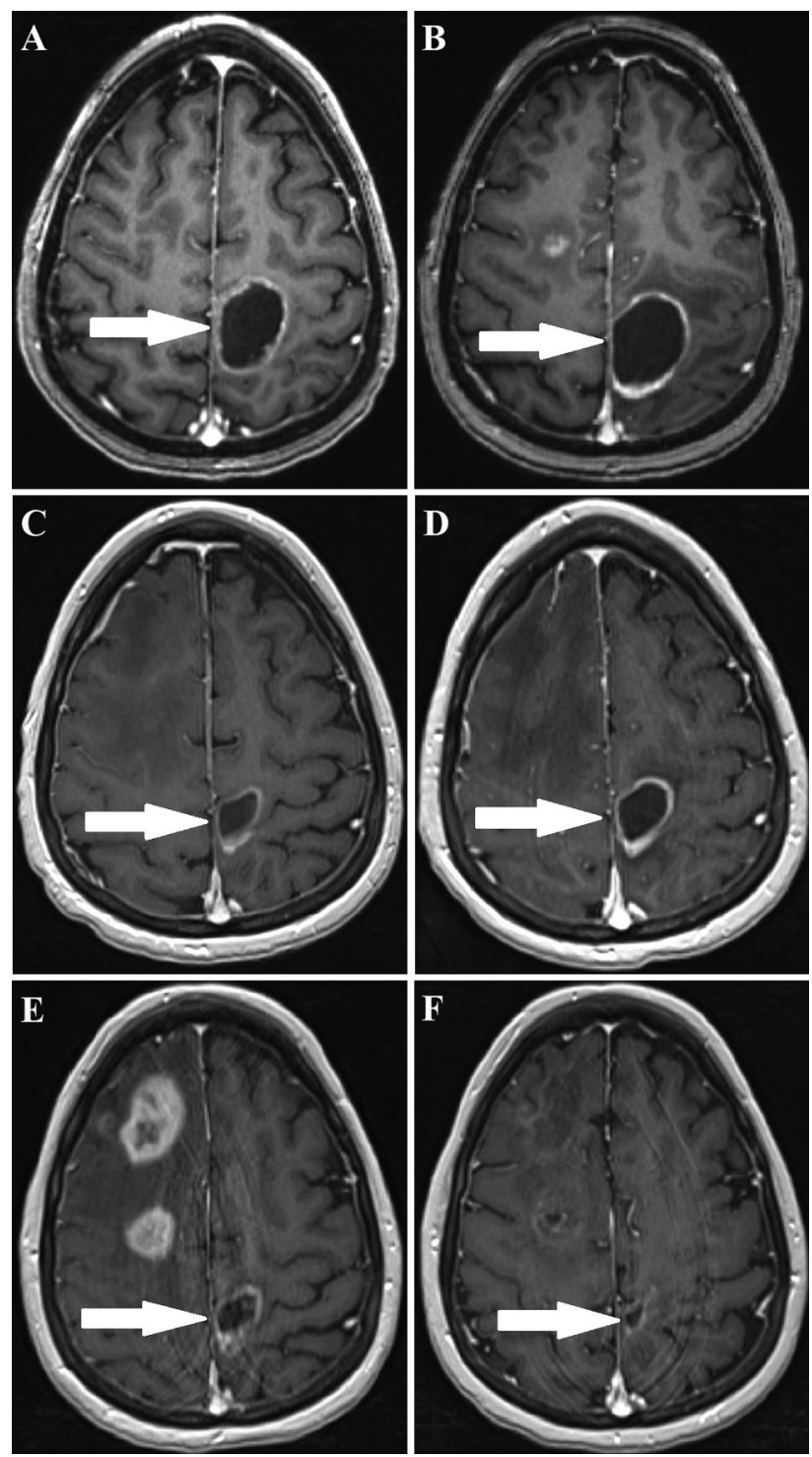

Figure 1. Left parietal lesion treated with two stages of 15 Gy one month apart. A: Prior to stage one. B: Prior to stage two. C: Three months after stage one. D: Four months after stage one. E: Six months after stage one. F: Eight months after stage one.

\section{Discussion}

For small lesions, stereotactic radiosurgery provides both excellent local control rates and minimal risk of toxicity $(2,5)$. The former is due to the high dose administered while the latter is due to the small volume treated. Unfortunately, larger lesions require a larger volume treated so the historical approach to minimize the risk of toxicity was to decrease the dose. In the seminal dose-escalation study for SRS, risk of chronic grade 3-5 neurotoxicity was $10 \%$ for tumors $\leq 20 \mathrm{~mm}$ that received 

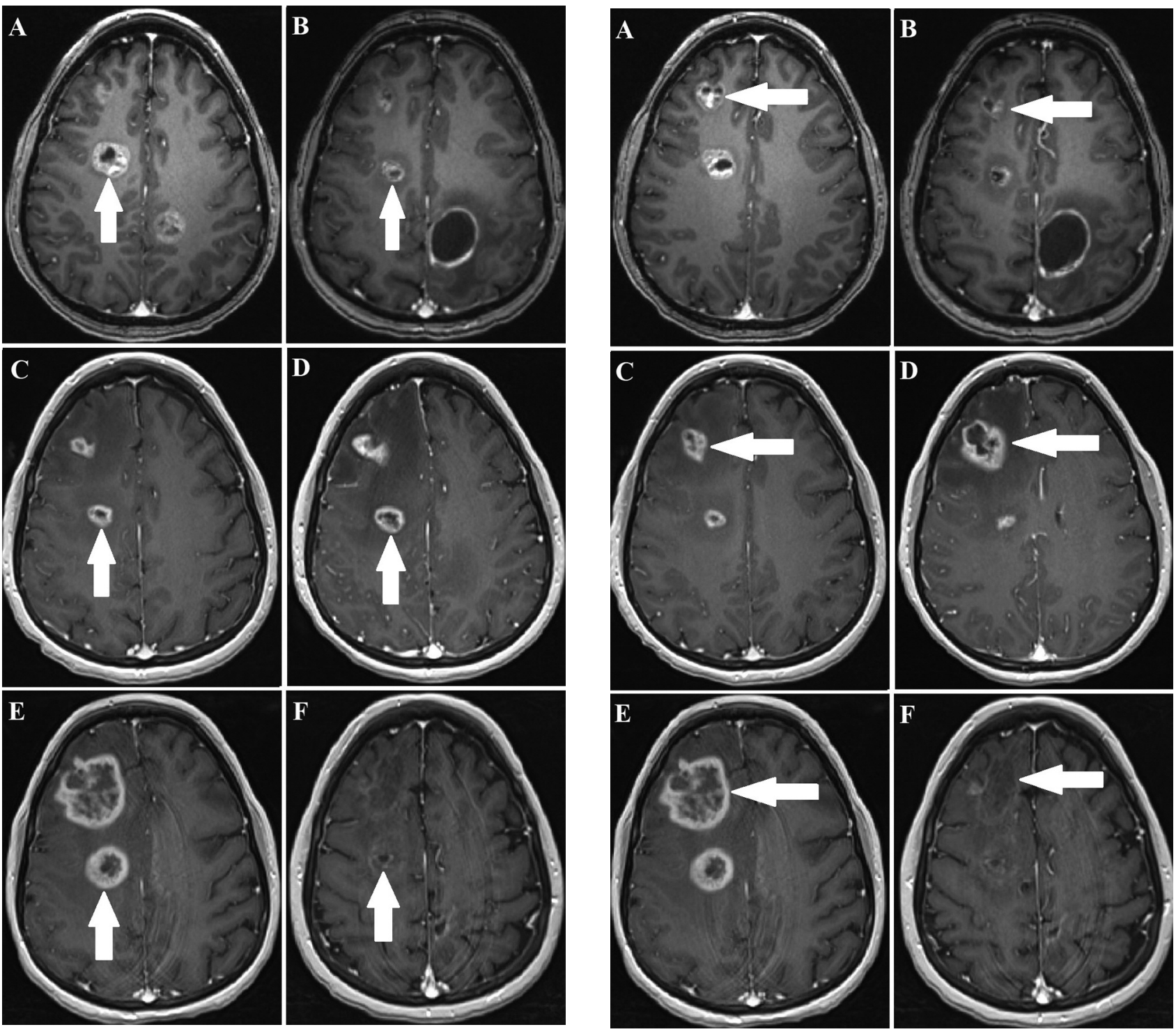

Figure 2. Posterior right frontal lesion treated with 18 Gy in one fraction. A: Prior to treatment. B: One month after treatment. C: Three months after treatment. D: Four months after treatment. E: Six months after treatment. F: Eight months after treatment and after receiving bevacizumab.

$24 \mathrm{~Gy}, 20 \%$ for tumors $21-30 \mathrm{~mm}$ that received $18 \mathrm{~Gy}$, and $14 \%$ for tumors $31-40 \mathrm{~mm}$ that received $15 \mathrm{~Gy}$ (2). While dose reduction for larger lesions successfully mitigates toxicity to a more acceptable level, it hampers local tumor control rates based on fundamental radiobiology principles indicating larger tumors with more cells require a higher dose to achieve the same level of tumor control compared to smaller tumors with fewer cells (6). Accordingly, while lesions $\leq 20 \mathrm{~mm}$ receiving 24 Gy have a 1-year local control rate of $85 \%$, tumors $21-30$
Figure 3. Anterior right frontal lesion treated with $18 \mathrm{~Gy}$ in one fraction. A: Prior to treatment. B: One month after treatment. C: Three months after treatment. D: Four months after treatment. E: Six months after treatment. F: Eight months after treatment and after receiving bevacizumab. $\mathrm{mm}$ receiving 18 Gy have a 1 year local control rate of $49 \%$ and tumors $31-40 \mathrm{~mm}$ receiving 15 Gy have a 1 year local control rate of $45 \%$ (5).

An alternative approach to dose de-escalation in larger lesions is to fractionate the treatment, as this may allow for local control while limiting toxicity. From a radiobiological perspective, fractionation reduces toxicity since it provides time for normal tissue repair (7). For brain metastasis $>2 \mathrm{~cm}$, Minniti et al. retrospectively compared 15-18 Gy in one 
fraction to $27 \mathrm{~Gy}$ in 3 fractions (i.e. FSRS) and showed oneyear local control rates improved from $77 \%$ to $91 \%$ and rate of toxicity decreased from $20 \%$ to $8 \%$ (3). Similarly, Angelov et al. showed that brain metastasis $>2 \mathrm{~cm}$ treated with a median $30 \mathrm{~Gy}$ in 2 equal stages delivered a median of 34 days apart (i.e. SSRS) had a six-month local control rate of $88 \%$ with an $11 \%$ rate of adverse effects (4).

While the improved toxicity profile with FSRS and SSRS is likely due to lower dose per fraction, the improvement in local control may be explained by increased BED delivered to the disease. Doses of $15 \mathrm{~Gy}$ and $18 \mathrm{~Gy}$ in 1 fraction have a respective $\mathrm{BED}$ of $37.5 \mathrm{~Gy}$ and $50.4 \mathrm{~Gy}$ assuming an alpha/beta ratio of 10 . In contrast, $27 \mathrm{~Gy}$ in 3 fractions ( $9 \mathrm{~Gy}$ per fraction) has a BED of $51.3 \mathrm{~Gy}$ and $30 \mathrm{~Gy}$ given in 2 fractions (15 Gy per fraction) has a BED of $75 \mathrm{~Gy}$. The major caveat for the latter is that BED calculations are not designed to incorporate the length of the time interval between the two fractions. Regardless, there is certainly a greater BED delivered to the tumor with multi-fraction regimens which likely explains the improved local control.

It is unclear what size threshold should be used to determine which lesions should be treated with a single fraction compared to which lesions should be treated with either FSRS or SSRS. The presented patient had a $3.3 \mathrm{~cm}$ lesion treated with SSRS that did not develop radionecrosis but two lesions $2.1 \mathrm{~cm}$ and $2.3 \mathrm{~cm}$ in size treated in a single fraction did develop symptomatic radionecrosis refractory to steroids but responsive to bevacizumab. While her treatment followed current national guidelines which stipulate that lesions $>3 \mathrm{~cm}$ should be treated in multiple fractions, our findings along with those of Minniti et al. and Angelov et al. instead suggest that all brain metastasis $>2 \mathrm{~cm}$ should be treated with a more fractionated approach (1, 3-4).

The presented patient had two punctate lesions that were adequately treated in a single fraction without toxicity. Because V12 Gy $>10 \mathrm{cc}$ of normal brain is considered the general radionecrosis threshold, there is no expected benefit with a multi-fraction approach for such very small lesions $(8,9)$. It is noteworthy that size is not a sole predictor of radionecrosis and other factors such as tumor location, conformality indices, and heterogeneity indices also have an important role $(8,10-12)$. Therefore, certain lesions otherwise $\leq 2 \mathrm{~cm}$ may also benefit from FSRS/SSRS. While further studies are necessary to delineate clear indications for FSRS/SSRS in tumors $\leq 2 \mathrm{~cm}$, until then we recommend strong consideration for using FSRS/SSRS when in doubt since there appears to be no major detriment.

\section{Conclusion}

We described a patient with a $3.3 \mathrm{~cm}$ lesion treated with SSRS that did not develop radionecrosis, and two lesions $2.1 \mathrm{~cm}$ and $2.3 \mathrm{~cm}$ in size which did develop symptomatic radionecrosis from single fraction SRS refractory to steroids but responsive to bevacizumab. Congruent with other recent studies, our report suggests that large brain metastasis be considered for FSRS/SSRS.

\section{Conflicts of Interest}

The Authors report no conflicts of interest in relation to this study.

\section{Authors' Contributions}

Neil Chevli: Data curation, validation, review \& editing. Hui-Chuan Wang: Conceptualization, review \& editing. Prachi Dubey: Conceptualization, review \& editing. Waqar Haque: Conceptualization, investigation, review \& editing. Andrew M. Farach: Conceptualization, review \& editing. Ramiro Pino: Conceptualization, review \& editing. Robert C. Rostomily: Conceptualization, review \& editing. E. Brian Butler: Conceptualization, investigation, review \& editing. Bin S. Teh: Conceptualization, investigation, project administration, supervision, validation, review \& editing.

\section{References}

1 NCCN Guidelines Central Nervous System Cancers Version 3.2020. Available at: https://www.ncen.org/professionals/ physician_gls/pdf/cns.pdf [Last accessed on December 3, 2020]

2 Shaw E, Scott C, Souhami L, Dinapoli R, Kline R, Loeffler J and Farnan N: Single dose radiosurgical treatment of recurrent previously irradiated primary brain tumors and brain metastases: final report of RTOG protocol 90-05. Int J Radiat Oncol Biol Phys 47(2): 291-298, 2000. PMID: 10802351. DOI: 10.1016/s0360-3016(99)00507-6

3 Minniti G, Scaringi C, Paolini S, Lanzetta G, Romano A, Cicone F, Osti M, Enrici RM and Esposito V: Single-Fraction versus multifraction $(3 \times 9 \mathrm{~Gy})$ stereotactic radiosurgery for large $(>2$ $\mathrm{cm}$ ) brain metastases: A comparative analysis of local control and risk of radiation-induced brain necrosis. Int J Radiat Oncol Biol Phys 95(4): 1142-1148, 2016. PMID: 27209508. DOI: 10.1016/j.jirobp.2016.03.013

4 Angelov L, Mohammadi AM, Bennett EE, Abbassy M, Elson P, Chao ST, Montgomery JS, Habboub G, Vogelbaum MA, Suh JH, Murphy ES, Ahluwalia MS, Nagel SJ and Barnett GH: Impact of 2-staged stereotactic radiosurgery for treatment of brain metastases $\geq 2 \mathrm{~cm}$. J Neurosurg 129(2): 366-382, 2018. PMID: 28937324. DOI: $10.3171 / 2017.3 . J N S 162532$

5 Vogelbaum MA, Angelov L, Lee SY, Li L, Barnett GH and Suh $\mathrm{JH}$ : Local control of brain metastases by stereotactic radiosurgery in relation to dose to the tumor margin. J Neurosurg 104(6): 907912, 2006. PMID: 16776334. DOI: 10.3171/jns.2006.104.6.907

6 Puck TT, Morkovin D, Marcus PI and Cieciura SJ: Action of xrays on mammalian cells. II. Survival curves of cells from normal human tissues. J Exp Med 106(4): 485-500, 1957. PMID: 13475608. DOI: 10.1084/jem.106.4.485

7 Thames HD Jr, Withers HR, Peters LJ and Fletcher GH: Changes in early and late radiation responses with altered dose fractionation: implications for dose-survival relationships. Int J Radiat Oncol Biol Phys 8(2): 219-226, 1982. PMID: 7085377. DOI: $10.1016 / 0360-3016(82) 90517-x$ 
8 Korytko T, Radivoyevitch T, Colussi V, Wessels BW, Pillai K, Maciunas RJ and Einstein DB: 12 Gy gamma knife radiosurgical volume is a predictor for radiation necrosis in non-AVM intracranial tumors. Int J Radiat Oncol Biol Phys 64(2): 419-424, 2006. PMID: 16226848. DOI: 10.1016/j.ijrobp.2005.07.980

9 Blonigen BJ, Steinmetz RD, Levin L, Lamba MA, Warnick RE and Breneman JC: Irradiated volume as a predictor of brain radionecrosis after linear accelerator stereotactic radiosurgery. Int J Radiat Oncol Biol Phys 77(4): 996-1001, 2010. PMID: 19783374. DOI: 10.1016/j.ijrobp.2009.06.006

10 Nedzi LA, Kooy H, Alexander E 3rd, Gelman RS and Loeffler JS: Variables associated with the development of complications from radiosurgery of intracranial tumors. Int J Radiat Oncol Biol Phys 21(3): 591-599, 1991. PMID: 1907957. DOI: 10.1016/03603016(91)90675-t
11 Valéry CA, Cornu P, Noël G, Duyme M, Boisserie G, Sakka LJ, Mazeron JJ and van Effenterre R: Predictive factors of radiation necrosis after radiosurgery for cerebral metastases. Stereotact Funct Neurosurg 81(1-4): 115-119, 2003. PMID: 14742974. DOI: $10.1159 / 000075114$

12 South M, Teh BS, Crane M and dela Paulino AC: Periventricular spreading necrotic lesions after stereotactic radiosurgery. Poster presented at: Radiological Society of North America Annual Meeting; November 30-December 5, 2008; Chicago, IL, USA.

Received January 20, 2021

Revised February 1, 2021

Accepted February 2, 2021 